\title{
MATHEMATICAL MODELING OF PHYSICAL PROPERTIES OF ANISOTROPIC MATERIALS
}

\author{
${ }^{1}$ Yuriy BELOKON, ${ }^{2}$ Aleksander YAVTUSHENKO, ${ }^{3}$ Victor PROTSENKO, ${ }^{4}$ Yulia BONDARENKO, \\ ${ }^{5}$ Andrii CHEILYTKO
}

\author{
1,2,3,4,5Zaporizhzhia National University, Zaporizhzhia, Ukraine, ${ }^{1}$ belokon.zp@gmail.com, \\ 2ayav2017@gmail.com, ${ }^{3}$ protsenkovm@yahoo.com, ${ }^{4}$ uvbondarenko.1984@gmail.com, \\ 5uvbondarenko.1984@gmail.com
}

https://doi.org/10.37904/metal.2020.3500

\begin{abstract}
The problem of selecting a material with an extreme value of its performance using its anisotropy is considered. It is important for specialists of metallurgical profile to be able not only to select the material for realization of the set engineering task, but also to use its anisotropy, and to be able to determine the orientation of the material with the extreme value of its performance. Mathematical modeling and computer analysis of anisotropy of tensor coefficients using the example of thermal expansion coefficient have been performed. Since thermal expansion, like any tensor physical property of crystals, is a continuous function of direction, then in order to determine the directions with a zero value of thermal expansion, the following ratio must be satisfied: $\alpha_{n}=0$. This can only happen if the main components of the thermal expansion tensor have different symbols. The Mathcad Prime 6 software complex has defined a function that performs the calculation of the value of thermal expansion coefficients in crystals in any direction, calculated the value and position of extremums of thermal expansion coefficients, and constructed an index surface, a stereographic projection of the index surface and the cross section of the index surface of thermal expansion coefficients $X_{1} X_{3}$. The lowest and highest values of the thermal expansion coefficient of the crystal have been found.
\end{abstract}

Keywords: Anisotropy, tensor, vector, texture, index projection, stereographic projection, orientation

\section{INTRODUCTION}

In the study of the theory of plastic flow of solids, one of the main goals is to master ability to perform mathematical modeling and computer analysis of anisotropy of tensor coefficients [1-3], which define the work of mono- and polycrystalline materials in different conditions and modes; and to determine the choice of materials with optimal characteristics for specific scientific and engineering solutions. For specialists of metallurgical profile it is important to be able not only to select the material for the realization of the set engineering task, but also to use the anisotropy to determine the orientation of the material with the extreme value of its performance.

In this regard, the solution of extreme problems, implemented both analytically and numerically by using modern computer programs, the Mathcad Prime 6 software package in particular, appears to be an urgent task. This allows to comprehensively study the behavior of the material in the process of its development and processing; analyze the geometry; perform engineering calculations; obtain photorealistic images; check models without testing experimental samples and save the researcher's time [2].

If the properties of sample cut from a material do not depend on its orientation, the material is called isotropic. Otherwise, the material is called anisotropic. Depending on what criterion is taken when identifying the properties of the specimens, it could mean mechanical, optical, thermal and other types of anisotropy. Crystals, for example, are always anisotropic, which is determined by their internal structure, since the atoms in the crystal cell are arranged in a very specific way. Knowing the structure of the crystal cell, we can draw some 
conclusions about the nature of the anisotropy, for example, indicate symmetry planes. Samples cut from the crystal symmetrically with respect to such a plane will reveal identical properties. Technical alloys consist of crystalline grains, the orientation of which is random and arbitrary. Therefore, in a body consisting of a large number of such grains, it is impossible to indicate any preferred direction that differs from others. Polycrystalline metal behaves, on average, as an isotropic body. In this case, it is assumed that the sample sizes are large enough and it contains quite a few crystalline grains. Small samples consisting of a small number of grains will exhibit different properties, but this difference is completely random, as it depends not on the orientation of sample, but on the random orientations of its constituent grains [4].

In rolled metal, the grains are deformed in the direction of rolling, and so-called texture is formed. Therefore, the properties of the samples cut in the rolling direction and in the transverse direction will be different. The same anisotropy occurs in virtually all types of metal processing by pressure. However, the anisotropy of the elastic properties associated with the presence of texture is small; the difference in the modulus of elasticity of the rods, the axes which are oriented in the rolling direction and in the transverse direction, may be neglected. However, the plastic properties for these directions, the elastic limit or the yield stress are already noticeably different. Proper thermal treatment of the deformed metal removes the anisotropy or at least reduces it $[5,6]$.

The anisotropy coefficient $\mathrm{K} \mathrm{L}$ is taken into account if the first main stress in bending or stretching - the compression is directed perpendicularly to the rolling direction of the metal (Table 1). Anisotropy is not taken into account during torsion $\left(\mathrm{K}_{\mathrm{L}}=1\right)$.

Table 1 Anisotropy coefficient values [7]

\begin{tabular}{|c|c|}
\hline Breaking point $\sigma_{\mathrm{B}}, \mathbf{M P a}$ & $\mathbf{K}$ \\
\hline Until 600 & 0.90 \\
\hline From 600 to 900 & 0.86 \\
\hline From 900 to 1200 & 0.83 \\
\hline Above 1200 & 0.80 \\
\hline
\end{tabular}

The anisotropy coefficient is taken into account when calculating the material endurance limit [5].

During the process of cold plastic deformation, mechanical energy is converted into thermal energy. It is noted that the steel strip in the center of deformation during rolling cannot be regarded as an isotropic material: the breaking point and mechanical properties of the metal along the length of the arc of contact vary [7].

The aim of this work is the development of an algorithm and program for calculating the tensor physical properties of a material, particularly the coefficient of thermal expansion in the Mathcad Prime 6 software complex; optimization of materials through understanding of the dependence of properties on the structure

\section{RESEARCH METHODOLOGY}

As an example, let us consider the problem of determining the thermal stability of a class 2 crystal under thermal expansion conditions [6].

Since the thermal expansion, like any tensor physical property of crystals, is a continuous directional function, then to determine the required directions with zero thermal expansion, the following relation must be satisfied $\alpha_{n}=0$. This can only happen if the main components of the thermal expansion tensor have different signs.

The thermal expansion of crystals is described by a symmetric second-rank tensor $\left[\alpha_{i j}\right\rfloor$. The magnitude of thermal expansion in an arbitrary direction $n\left(n_{1}, n_{2}, n_{3}\right)$ in the crystal is defined as $\alpha(n)=\alpha_{i j} n_{i} n_{j}$, or in matrix form (eqn 1): 


$$
\alpha(n)=\left(n_{1} n_{2} n_{3}\right)\left(\begin{array}{lll}
\alpha_{11} & \alpha_{12} & \alpha_{13} \\
\alpha_{12} & \alpha_{22} & \alpha_{23} \\
\alpha_{13} & \alpha_{23} & \alpha_{33}
\end{array}\right)\left(\begin{array}{l}
n_{1} \\
n_{2} \\
n_{3}
\end{array}\right)
$$

The value $\alpha(n)$ reaches the extreme values in the directions of the eigenvectors of the tensor $\left[\alpha_{i j}\right\rfloor$, and these extreme values are equal to the corresponding eigenvalues of the tensor $\left[\alpha_{i j}\right\rfloor$. If all eigenvalues of the tensor $\left\lfloor\alpha_{i j}\right\rfloor$ are positive (negative), then all $\alpha(n)$ are positive (negative). If there are both positive and negative values among the eigenvalues of the tensor $\left[\alpha_{i j}\right\rfloor$, then $\alpha(n)$ takes both positive and negative values; at the same time on some cone of directions $\alpha(n)=0$.

For class 2 crystals in the crystal-physical coordinate system in the standard setting $\left(X_{2} \| 2\right)$, the tensor $\left[\alpha_{i j}\right\rfloor$ has the following form (eqn. 2):

$$
\left[\alpha_{i j}\right]=\left[\begin{array}{ccc}
\alpha_{11} & 0 & \alpha_{13} \\
0 & \alpha_{22} & 0 \\
\alpha_{13} & 0 & \alpha_{33}
\end{array}\right]
$$

$$
\text { where } \begin{aligned}
\alpha_{11} & =12 \cdot 10^{-6}\left(K^{-1}\right) \\
\alpha_{22} & =44.8 \cdot 10^{-6}\left(K^{-1}\right) \\
& \alpha_{33}=32 \cdot 10^{-6}\left(K^{-1}\right) .
\end{aligned}
$$

We solve the problem in the Mathcad Prime 6 software complex [8]. We introduce the tensor of coefficients of thermal expansion of crystals - input of coefficients of thermal expansion of crystals (eqn. 3).

$$
\begin{aligned}
& \alpha_{11}:=12 \cdot 10^{-6} \quad \alpha_{22}:=44.8 \cdot 10^{-6} \quad \alpha_{33}:=32 \cdot 10^{-6} \quad \alpha_{13}:=-12 \cdot 10^{-6} \\
& \alpha:=\left[\begin{array}{ccc}
\alpha_{11} & 0 & \alpha_{13} \\
0 & \alpha_{22} & 0 \\
\alpha_{13} & 0 & \alpha_{33}
\end{array}\right]
\end{aligned}
$$

To calculate the eigenvalues and eigenvectors of the tensor $\left\lfloor\alpha_{i j}\right\rfloor$, we use the built-in eigenvalues and eigenvector functions - calculation of value and position of coefficient extrema of thermal expansion of crystals (eqn. 4).

$$
\begin{aligned}
& \lambda:=\text { eigenvals }(\alpha) \\
& \lambda=\left[\begin{array}{l}
4.48 \cdot 10^{-5} \\
3.762 \cdot 10^{-5} \\
6.38 \cdot 10^{-6}
\end{array}\right] \\
& n_{\text {max }}:=\operatorname{eigenvec}\left(\alpha, \lambda_{1}\right) \quad n_{\max }{ }^{\mathrm{T}}=\left[\begin{array}{lll}
0 & 1 & 0
\end{array}\right] \\
& n_{\text {min }}:=\operatorname{eigenvec}\left(\alpha, \lambda_{3}\right) \quad n_{\min }{ }^{\mathrm{T}}=\left[\begin{array}{lll}
0.906 & 0 & 0.424
\end{array}\right] \\
& \theta_{\min }:=\operatorname{acos}\left(n_{\min }\right) \cdot \frac{180}{\pi}=25.097
\end{aligned}
$$


To visualize the anisotropy of the coefficient of thermal expansion of crystals, we construct a function that, according to expression (1), conducts the calculation of the value of the coefficient of thermal expansion of crystals (in $10^{-6} \mathrm{~K}^{-1}$ ) in an arbitrary direction $n$ (eqn. 5).

$$
\alpha_{n}(\phi, \theta):=\left|\begin{array}{l}
n \leftarrow\left[\begin{array}{c}
\cos (\phi) \cdot \cos (\theta) \\
\sin (\phi) \cdot \cos (\theta) \\
\sin (\theta)
\end{array}\right] \\
n^{\mathrm{T}} \cdot \alpha \cdot n \cdot 10^{6}
\end{array}\right|
$$

\section{RESULTS OF THE STUDY}

In eqn. 6 the program for calculating the coefficient of thermal expansion in the software complex Mathcad Prime 6 is presented. The construction of the index surface of the coefficient of thermal expansion of crystals, its stereographic projection, as well as the cross section of the index surface with the plane $X_{1} X_{3}$ are presented respectively in Figures 1-3.

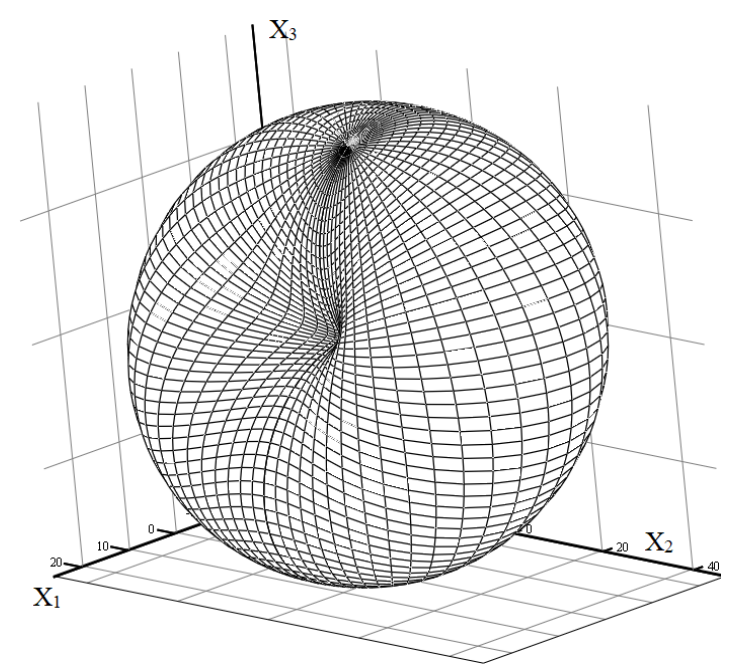

Figure 1 Index surface of coefficients of thermal expansion of crystals

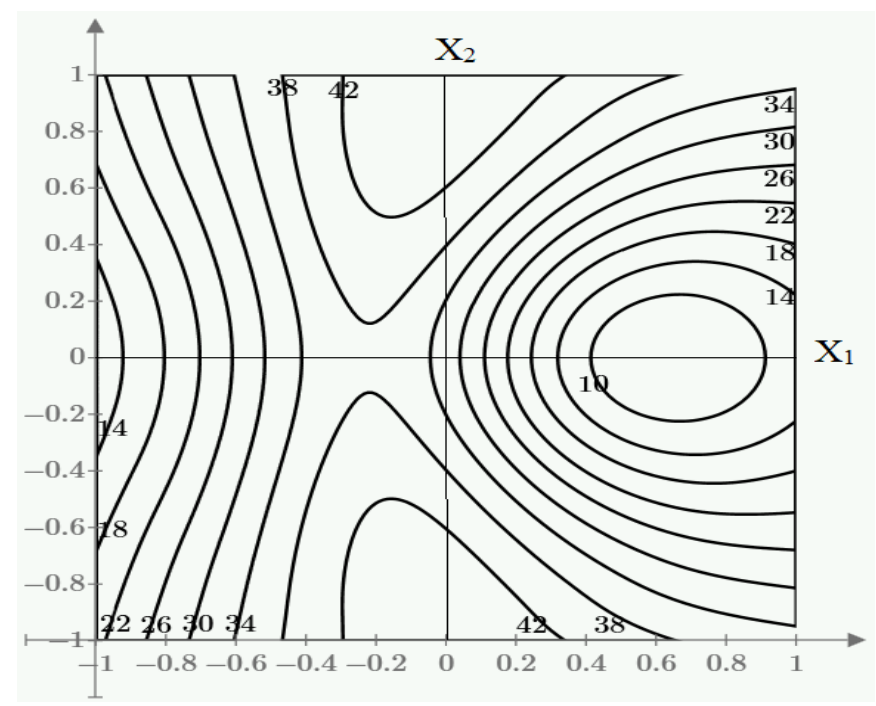

Figure 2 Stereographic projection of the index surface of the coefficients of thermal expansion of crystals (in $\left.10^{-6} \mathrm{~K}^{-1}\right)$ 


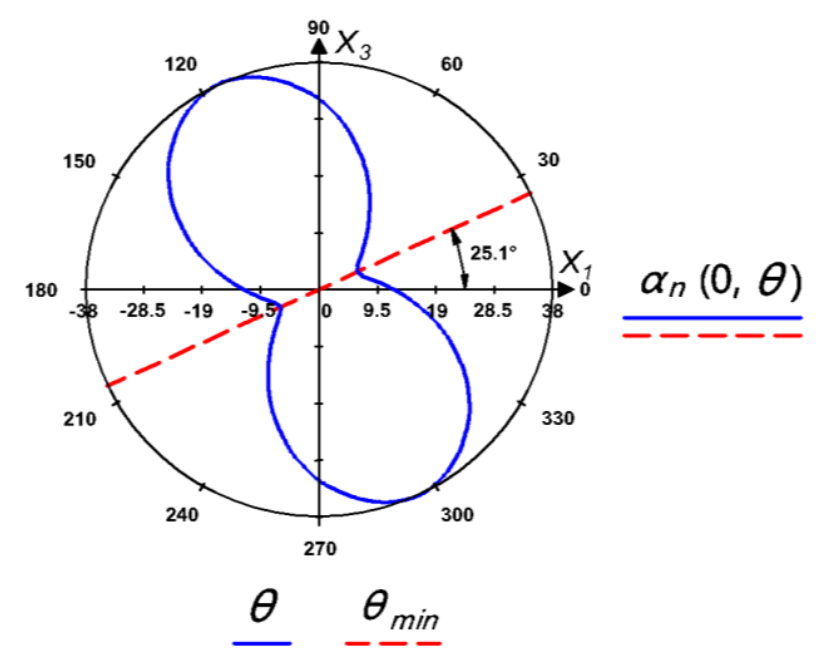

Figure 3 Cross section of the index surface of the coefficients of thermal expansion of crystals by the plane $X_{1} X_{3}$

Points $X_{1}$ and $X_{2}$ in Figure 1 are the outputs of the corresponding crystal-physical axes; the crystal-physical $X_{3}$ axis is directed upwards from the centre of the stereographic projection (see eqn. 6).

$$
\begin{aligned}
& \phi_{0}:=0 \quad \phi_{1}:=2 \cdot \pi \quad N_{\phi}:=61 \\
& \theta_{0}:=\frac{-\pi}{2} \quad \theta_{1}:=\frac{\pi}{2} \quad N_{\theta}:=61 \\
& f s(\phi, \theta):=\alpha_{n}(\phi, \theta) \cdot\left[\begin{array}{c}
\cos (\phi) \cdot \cos (\theta) \\
\sin (\phi) \cdot \cos (\theta) \\
\sin (\theta)
\end{array}\right] \quad S 1:=\text { CreateMesh }\left(f s, \phi_{0}, \phi_{1}, \theta_{0}, \theta_{1}, N_{\phi}, N_{\theta}\right) \\
& \phi_{0}:=0 \quad \phi_{1}:=2 \cdot \pi \quad N_{\phi}:=181 \\
& \theta_{0}:=0 \quad \theta_{1}:=\frac{\pi}{2} \quad N_{\theta}:=91 \\
& f c(\phi, \theta):=\left[\begin{array}{c}
\tan \left(\frac{\pi}{4}-\frac{\theta}{2}\right) \cdot \cos (\phi) \\
\tan \left(\frac{\pi}{4}-\frac{\theta}{2}\right) \cdot \sin (\phi) \\
\alpha_{n}(\phi, \theta)
\end{array}\right] \quad C 1:=\text { CreateMesh }\left(f c, \phi_{0}, \phi_{1}, \theta_{0}, \theta_{1}, N_{\phi}, N_{\theta}\right)
\end{aligned}
$$

Thus, based on the calculation results, it follows:

a) the smallest value of the coefficient of thermal expansion, equal to $6.4 \cdot 10^{-6} \mathrm{~K}^{-1}$, is observed in the direction lying at the plane $X_{1} X_{3}$ and making an angle of $25.1^{\circ}$ with the $X_{2}$ axis;

b) the highest value of the coefficient of thermal expansion, equal to $44.8 \cdot 10^{-6} \mathrm{~K}^{-1}$, corresponds to the direction of the axis $X_{2}(Y$ - section);

c) as it may be seen from the obtained values of the main coefficients of thermal expansion of the crystals, all of them are positive; therefore, there are no directions with zero value of thermal expansion coefficient in these crystals. 
As from eqn 4 follows, tensor $\left[\alpha_{i j}\right\rfloor$ in the system of its main axes $X_{1} X_{2} X_{3}$ has three different eigenvalues; the symmetry of the index surface of the coefficient of thermal expansion of the crystal - $m m m$, which is consistent with the Neumann principle [1].

\section{CONCLUSION}

An algorithm and a program for calculating the tensor physical properties of the material, particularly the coefficient of thermal expansion in the software complex Mathcad Prime 6, have been developed. The construction of the index surface of the coefficient of thermal expansion of crystals, its stereographic projection, as well as the cross section of the index surface by the plane $X_{1} X_{3}$ have been conducted. The smallest value of the coefficient of thermal expansion equal to $6.4 \cdot 10^{-6} \mathrm{~K}^{-1}$ has been found, which is observed in the direction lying in the plane $X_{1} X_{3}$ and constituting an angle of $25.1^{\circ}$ with the $X_{2}$ axis; and the highest value of the coefficient of thermal expansion, equal to $44.8 \cdot 10^{-6} \mathrm{~K}^{-1}$, corresponding to the direction of the axis $X_{2}$ ( $Y$ - section).

\section{REFERENCES}

[1] NEWNHAM, R.E. Properties of Materials: Anisotropy, Symmetry, Structure. Oxford: Oxford University Press, 2005.

[2] KOCKS, U.F., TOME, C.N., WENK, H.R. Texture and Anisotropy. Cambridge: Cambridge University Press, 1998.

[3] LOVETT, D.R. Tensor Properties of Crystals. Bristol: Institute of Physics Publishing, 1999.

[4] PANJKOVIC, V. Friction and the Hot Rolling of Steel. 2nd ed. Boca Raton, FL: CRC Press, 2014.

[5] BELOKON, Y., ZHEREBTSOV, A., BELOKON, K. The investigation of nanostructure formation in intermetallic $Y$ TiAl alloys. In: IEEE International Young Scientists Forum on Applied Physics and Engineering (YSF-2017). 2017. Lviv: IEEE, pp. 311-314.

[6] SEREDA, B., BONDARENKO, Y., SEREDA, D. Multicomponent saturing of titan alloys by SHS. In: Materials Science and Technology Conference and Exhibition. 2011. Columbus: MS\&T, pp. 1715-1720.

[7] SEREDA, B., KRUGLYAK, I., KOVALENKO, A., SEREDA, D., VASILCHENKO, T. The deformation zone geometrical factors and its influence on deformation shift degree for the axial zone of rolled high bars. Metallurgical and Mining Industry. 2011, vol. 3, no. 7, pp. 102-106.

[8] OCHKOV, V.W. Mathcad 14 for Students, Engineers, and Designers. St. Petersburg: BHV Publisher House, 2007. 